Acta Cryst. (2002). A58 (Supplement), C127

STRUCTURE \& CONFORMATION OF SUBSTITUTED DIBENZO [d,g] [1,3,2]\&[1,3,6,2] DIOXA \& DIOXATHIAPHOSPHOCIN 6-OXIDES M. Krishnaiah N Jagadeesh Kumar

Sri Venkateswara University Physics Dept. of Physics S.V.University TIRUPATI ANDHRA PRADESH 517502 INDIA

Organophosphorus compounds are ubiquitous in nature, but suitably substituted phosphoryl unit has to be present in the molecule to exhibit significant physiological activity. Ring formation with phosphoryl unit can have profound effect on hydrolysis rate which can be adjustable for preferential hydrolysis by either ring size or exocyclic ester bonds. These compounds are often importance in terms of multiple application as insecticides, bactericides, fungicides and also in polymer and oil industries as stabilizer and antioxidants. Some of these families were evaluated for toxicity in the insect P-American. The structural investigations on a series of compounds have been studied to understand the conformation and identify the biological active phosphorus moieties. The present study is devoted to (I) 6-(4-methyl anrloxy)-120X0dibenzo [d,g][1,3,2] dioxaphosphocin oxide (II) 1,2,4,8,10,11-hexachloro-6trichloromethyl 12H-dibenzo [d,g] [1,3,2] dioxaphophocin 6-oxide, (III) 6bis92-chloroethyl) amino 2,10-dichloro 12-trichlormethyl 12H-dibenxo [d,g] [1,3,2] dioxaphosphocin 6-oxide, (IV) 2,10 dichlo 6-trichloromethyl dibenzo [d,g] [1,3,6,2] dioxathiaphosphocin, (V) 6-bis (2-chloroethyl) amino 2,10 dichlorodibenzo $[\mathrm{d}, \mathrm{g}][1,3,6,2]$ dioxathiaphosphocin 6-oxide. The solid state structure of the molecular reveal that the conformation for the eight membered dioxaphophocin ring is a distorted boat (DB) in I, II \& III and for the dioxathiaphosphocin ring is twist boat (TB) in III and boat-chair (BC) in IV. The effect of the substitutes on the conformation and geometrical parameters are compared with other corresponding ones reported and their biological activity.

Keywords: ORGANOPHOSPHORUS COMPOUNDS, DIOXAPHOSPHOCIN RINGS, 8-MEMBERED HETEROCYCLES

Acta Cryst. (2002). A58 (Supplement), C127

\section{INTRAMOLECULAR RESONANCE ASSISTED HYDROGEN BOND AND AGGREGATION OF 2-QUINOLONES: A NEUTRON DIFFRACTION STUDY}

G. Punte $^{1}$ G. Pozzi ${ }^{1}$ A. E. Goeta ${ }^{2}$ C. Wilson ${ }^{3}$ G. Romanelli ${ }^{4}$ J. C. Autino ${ }^{4}$ IFLP And LANADI Departamento De F/'isica. UNLP Cc 67 LA PLATA 1900 ARGENTINA

${ }^{1}$ LANADI e IFLP. Departamento de Fl'isica, Facultad de Ciencias Exactas, Universidad Nacional de La Plata, CC 67, (1900), La Plata, Argentina. ${ }^{2}$ Department of Chemistry, University of Durham, Durham, DH1 3LE, United Kingdom ${ }^{3}$ ISIS, Rutherford Appleton Laboratories, Chilton, Didcot, Oxon, OX11 0QX, United Kingdom. ${ }^{4}$ LADECOR, Dep. de Qul'imica, Fac. de Ciencias Exactas, Universidad Nacional de La Plata, 47 y 115, (1900), La Plata, Argentina

The nature of $\pi-\pi$ stacking interactions and their capability to be used as recognition elements are still open questions. The strength of these interactions seems to be influenced by the extension of the delocalization and the presence of heteroatoms. 4Hydroxy-2-quinolones derivatives with appropriate substituents at C3 have shown extended electron delocalization through resonance assisted hydrogen bonds (RAHB). Furthermore, various derivatives of N-alkyl-4-hydroxy-3-ethoxycarbonyl2-quinolones present diverse biological activities. For all these reasons, this family of compounds is interesting not only to disclose the competitive influence of the intramolecular interactions and the different cohesive forces at play but also to help towards the understanding of the mechanisms involved in their biological action. To this end, and as part of a more general project, we have performed a single crystal neutron diffraction study of 3-ethoxycarbonyl-4-hydroxy-6-methoxymethyleneoxy1-methyl-2-quinolone at $100 \mathrm{~K}$. It yields a molecular model that shows an intramolecular RAHB and delocalization beyond the fusioned rings and the resonant fragment. Packing analysis indicates that stacking interactions and $\mathrm{C}-\mathrm{H}$... hydrogen bonds (HBs) rule the three dimensional structure. Stacking originates centrosymmetric dimers, but bulky substituents at 3 and 6 prevent an infinite stacking arrangement. Dimers form ribbons along a through C-H... $\mathrm{HB}$ interactions. These ribbons are assembled in layers perpendicular to c. Dimers in adjacent layers, and so the layers themselves, are connected by $\mathrm{C}-\mathrm{H}$... $\mathrm{O} \mathrm{HBs}$ and a $\mathrm{C}(\operatorname{aryl})-\mathrm{H} . . \pi$ interaction which induce dimers belonging to adjacent layers to have their mean planes perpendicular to [011] and [0-11] respectively. Possible cooperativity of the interactions is discussed.
Acta Cryst. (2002). A58 (Supplement), C127

3-[(4-HYDROXY-5,8-DIMETHYL-2-OXO-2H-CHROMEN-3-YL)-4METHOXYPHENYLMETHYL]-5,8-DIMETHYL-2-OXO-2H-

CHROMEN-4-OLATE

L. Vijayalakshmi ${ }^{1}$ V. Parthasarathi ${ }^{2}$ A. Shah $^{3}$ V. Vora ${ }^{3}$ B. Desai ${ }^{3}$

${ }^{1}$ Department of Physics, Arignar Anna Government Arts College, Musiri-621 201. India. ${ }^{2}$ Bharathidasan University D-10, Sixth 'a' Cross, Western Extension, Thillainagar TIRUCHIRAPALLI TAMILNADU 820018 INDIA ${ }^{3}$ Department of Chemistry, Saurashtra University, Rajkot-360 005, India.

The structures of a large number of HIV-1 integrase inhibitors have in common two aryl units separated by a central linker and one of these aryl moieties must contain dihydroxy substituents in order to exhibit high inhibitory potency. Inclusion of the phenyl ring (similar to the title compound) in the horizontal dimer exhibited 2 -fold better inhibitory potency than the corresponding vertical dimer. In the present investigation, the structure determination was carried out to study the possible structure activity relationship.

The title compound $\mathrm{C}_{30} \mathrm{H}_{26} \mathrm{O}_{7}$ crystallizes in the orthorhombic space group $P 2_{1} 2_{1} 2_{1}$ with two molecules in the asymmetric unit with $\mathrm{a}=11.039(1) ; \mathrm{b}=$ 15.599 (2), $\mathrm{c}=28.704(4) \AA ; \mathrm{V}=4942.92(10) \AA^{3}, \mathrm{Z}=8$, wR $=0.164$ and final $\mathrm{R}=0.064$ for 3130 observations. In both the molecules, the coumarin rings are individually planar. All principal bond angles about methylene carbon in both the molecules are widened over normal tetrahedral values. Steric crowding about the methylene carbon may be responsible for this feature. The atoms C31and C33 of the methyl and methoxy groups in both the molecules are found to be disordered. The exocyclic bond angles about $\mathrm{C} 3$ and $\mathrm{C} 13$ differ in molecule A and molecule B. This asymmetry may arise from the effects of steric crowding within the molecule or from packing constraints. The repulsive forces between the non-bonded atoms might have been relieved by the rotation of the phenyl ring. The 4-hydroxycoumarins are intramolecularly hydrogen bonded between hydroxyls and carbonyls in both the molecules.

\section{Keywords: INTEGRASE INHIBITOR HYDROGEN BONDING} STERIC CROWDING

\section{Acta Cryst. (2002). A58 (Supplement), C127 ORGANIC ELECTRONIC MATERIALS \\ T. Siegrist C. Kloc J.H. Schoen}

Bell Laboratories 600 Mountain Avenue MURRAY HILL NJ 07974 USA

Recent successes in achieving very high mobilities in simple organic acene and related systems opened new areas in possible applications. In particular, refined crystal growth methods produced materials with unexpected properties at low temperatures. In particular, the vapor phase growth of organic crystals at elevated temperatures reduces the impurity levels while giving high crystal quality. Due to the higher growth temperatures, polymorphic differences between molecular packings are observed. In general, slightly denser molecular packings are seen for crystal growth at higher temperature. Correspondingly, measured charge carrier mobilities are higher in the denser packings.

Keywords: ORGANIC TRANSISTOR ELECTRONIC PROPERTIES 